\title{
Evaluación de los principales factores de rendimiento en gimnasia rítmica. Comparación entre diferentes niveles
}

\author{
Assessment of the main performance factors in rhythmic gymnastics. \\ Comparation between different levels \\ Carmen Ruano Masiá ${ }^{1}$, Roberto Cejuela Anta ${ }^{2}$ \\ 1 Centro de Investigación del Deporte. Universidad Miguel Hernández, Elche. España. \\ 2 Área de Educación Física y Deportiva. Departamento Didáctica General y Didácticas Específicas. Universidad de Alicante. España. \\ CORRESPONDENCIA: \\ Carmen Ruano Masiá \\ carmen.ruano@goumh.umh.es \\ Recepción: febrero 2018 • Aceptación: julio 2019 \\ CÓMO CITAR EL ARTÍCULO: \\ Ruano-Masiá, C., \& Cejuela-Anta, R. (2020). Evaluación de los \\ principales factores de rendimiento en gimnasia rítmica. Com- \\ paración entre diferentes niveles. Cultura, Ciencia y Deporte, \\ 15(44), 65-175.
}

\section{Resumen}

El objetivo de este estudio fue comparar los factores de rendimiento de la gimnasia rítmica estudiados en la literatura entre dos grupos de gimnastas individuales españolas de diferentes niveles (primera y tercera división nacional). 18 gimnastas (14.1 1 1.2 años) fueron evaluadas y divididas en dos grupos: élite $(n=4)$ y no élite $(n=14)$. Se realizaron test antropométricos, físicos (flexibilidad y capacidad de salto) y fisiológicos (frecuencia cardíaca y lactato). Las gimnastas del grupo élite mostraron menores valores antropométricos (sumatorio de pliegues cutáneos y porcentaje de grasa corporal). Además, se encontraron diferencias significativas en el índice de reactividad $(p<0.001)$ y en la flexibilidad en las articulaciones de hombro y cadera $(p<0.05$ y $p<0.01$, respectivamente), entre otros tests. Respecto a la frecuencia cardíaca no se encontraron grandes diferencias entre ambos grupos, mientras que las gimnastas élite presentaron valores superiores de lactato al finalizar el ejercicio, pero mostrando una mejor recuperación de este. Por tanto, podemos concluir que los valores antropométricos, la capacidad de salto, la flexibilidad y la capacidad de reciclaje del lactato serían los principales factores de rendimiento a evaluar para diferenciar a las gimnastas en función de su nivel. Estos factores medibles podrían utilizarse como identificadores del rendimiento deportivo, ayudando a la elaboración de los programas de entrenamiento por parte de los entrenadores

Palabras clave: Gimnastas, flexibilidad, antropometría, salto, frecuencia cardíaca, concentración de lactato

\begin{abstract}
The aim of this study was to compare the rhythmic gymnastics performance factors reported in the previous literature between two groups of Spanish individual gymnasts competing at different levels (first and third national division). 18 gymnasts (14.1 \pm 1.2 years) were measured, and divided into two groups, elite $(n=4)$ and non-elite $(n=14)$. The subjects underwent anthropometric, physical (jumping ability and flexibility) and physiological (heart rate and blood lactate) tests. Elite gymnasts showed lower anthropometric values (skinfolds addition and body fat percentage). In addition, significant differences were found in the reactivity index $(p<0.001)$, and in flexibility in shoulders and hips $(p<0.05$ and $p<0.01$, respectively). Respect to heart rate, no differences were found between both groups, while elite gymnasts showed higher lactate values at the end of the routine, but showing a better recovery. Thus, we can conclude that anthropometric values, jumping capacity, flexibility and lactate recycling capacity could be the main performance factors used to classify gymnasts according to their level. These measurable factors could be used as performance indicators, helping coaches to elaborate specific training programs.
\end{abstract}

Key words: Gymnasts, flexibility, anthropometry, jump, heart rate, lactate concentration 


\section{Introducción}

La Gimnasia Rítmica es un deporte olímpico femenino desde los Juegos Olímpicos de Los Ángeles 1984. Existen dos modalidades, individual y conjuntos, no obstante, en el presente estudio nos vamos a centrar en la primera. En ella, la gimnasta deberá realizar uno o varios ejercicios (en función del nivel) de 1 min $15 \mathrm{~s}$ a 1 min $30 \mathrm{~s}$ de duración con algunos de los 5 implementos reconocidos por la Federación Internacional de Gimnasia (FIG): cuerda, pelota, aro, mazas y cinta, en un tapiz de $13 \times 13 \mathrm{~m}$. Este ejercicio deberá contar de una serie de elementos obligatorios tanto corporales como con el aparato, y será evaluado por un jurado que, de acuerdo a un código de puntuación, valorará la precisión en los movimientos, la expresión, la originalidad y la coordinación con la música (Féderation Internationale de Gimnastique [FIG], 2017).

Se trata de un deporte de especialización temprana, que comienza sobre los 6-8 años. No obstante, durante la adolescencia (12-16 años), las gimnastas se encuentran en el momento decisivo de su carrera deportiva, ya que es en ese momento en el que se desarrollarán los factores determinantes del rendimiento (Benardot \& Czerwinski, 1991).

Nos encontramos ante una modalidad multifactorial, siendo varios los factores de rendimiento descritos en la literatura científica. Según Douda, Toubekis, Avloniti y Tokmakidis (2008), el componente antropométrico es uno de los mayores indicadores de rendimiento, ya que puede facilitar o dificultar la ejecución de los movimientos. Existen ciertas variables antropométricas que han sido relacionadas directamente con el rendimiento en competición, como la estatura, la circunferencia del muslo o el porcentaje de masa grasa (Douda et al., 2008; Purenović-Ivanović \& Popović, 2014), y otras que se han relacionado con otros factores de rendimiento: la capacidad de salto y la flexibilidad (Hume et al., 1993).

Por lo que a los factores físicos respecta, la Gimnasia Rítmica es un deporte en el que intervienen de manera combinada la fuerza explosiva o rápida, flexibilidad, coordinación, velocidad, equilibrio y reactividad o fuerza reactiva (capacidad para pasar de una fase excéntrica a concéntrica de manera rápida y aprovechando al máximo el ciclo estiramiento-acortamiento (Young, 1995)) teniendo todos ellos una importante influencia en el resultado final (Di Cagno et al., 2008a; Douda et al., 2008; Hume et al., 1993). Según Douda et al. (2008), la flexibilidad y la fuerza explosiva son los determinantes físicos principales del rendimiento. La primera cobra una importancia vital para ejecutar los elementos técnicos corporales, mientras que la segunda se materializa en la capacidad de salto, que puede ser uno de los indicadores de rendimiento útiles para la detección de talentos. Estas dos capacidades, sumadas a la reactividad del tren inferior y las características antropométricas suponen el $41 \%$ del éxito a la hora de realizar las dificultades corporales recogidas en el código de puntuación (Di Cagno et al., 2008a).

Las rutinas competitivas combinan durante 90 s elementos de muy alta intensidad con la manipulación de un implemento. Los movimientos que se realizan cambian muy rápido y se emplean todos los sistemas energéticos para satisfacer las demandas requeridas (Baldari \& Guidetti, 2001). No obstante, no se han reportado estudios recientes en los que se evalúen estas demandas en situaciones reales de competición. Según Guidetti, Baldari, Capranica, Persichini y Figura (2000), que realizaron un estudio de campo, el 49\% de la energía está provisto por la vía aeróbica, el $42 \%$ por la vía anaeróbica aláctica, y el 9\% viene de la vía anaeróbica láctica. Bien es cierto que es necesario tener en cuenta que las exigencias del código de puntuación en cuanto a los requerimientos técnicos del ejercicio van variando con los cambios en el mismo, por lo que estos porcentajes se podrían ver modificados.

La medida de lactato en sangre es una técnica poco empleada en este campo para la determinación de la intensidad del esfuerzo. Guidetti et al. (2000), registraron los niveles de este compuesto en gimnastas de élite, proponiendo que los ejercicios competitivos se realizan en torno al umbral láctico con todos los implementos salvo la cuerda, aparato que actualmente se ha eliminado del programa internacional de la FIG.

La gimnasia rítmica es un deporte creciente en España. En los últimos 10 años el número de licencias federativas ha aumentado en un $251 \%$ y el número de clubes en un 87,9\% (Consejo Superior de Deportes [CSD], 2016). En este país se están comenzando a conseguir buenos resultados en la modalidad de conjuntos, hecho que se constata con la plata conseguida en los Juegos Olímpicos celebrados en Río de Janeiro en 2016. No obstante, queda mucho camino por recorrer en la modalidad individual, donde las gimnastas españolas se encuentran bastante distanciadas de los primeros puestos a nivel internacional.

Encontramos publicaciones científicas en las que se analizan los factores de rendimiento de esta modalidad. En algunas de ellas, a pesar de estar publicadas en revistas de alto impacto y contar con metodologías validadas, no encontramos dos grupos de diferentes niveles entre los que se comparen los resultados de los tests realizados (Baldari \& Guidetti, 2001; Di Cagno et al., 2009, 2014; Guidetti et al., 2000; Romero, Palomino \& González, 2011). Por otra parte, existen artículos 
en los que sí podemos observar esa comparación (Di Cagno et al., 2008b; Donti, Bogdanis, Kritikou, Donti, \& Theodorakou, 2016; Douda et al., 2008; Hume et al., 1993), pero en ningún caso encontramos un estudio de estas características realizado con gimnastas españolas como muestra.

Por tanto, se plantea la necesidad de contrastar esos factores de rendimiento entre diferentes niveles dentro de la geografía española para conocer los aspectos decisivos para llegar a la élite, y así poder avanzar en el campo del entrenamiento deportivo y de la detección temprana de talentos, tan importante en este deporte, para así poder ser más competitivos a nivel internacional.

El objetivo del presente trabajo es comparar dos grupos de gimnastas españolas de diferentes niveles: Nacional Base (el inferior de los niveles de competición nacional) y Primera categoría (la máxima categoría nacional a excepción de la selección nacional), y establecer si existen diferencias entre ambos grupos, comparando con los factores de rendimiento antropométricos, físicos y fisiológicos estudiados en la literatura existente.

\section{Metodología}

\section{Participantes}

Diecisiete gimnastas femeninas $(n=17)$ de la modalidad individual participaron en el estudio (Edad media $14.1 \pm 1.2$ años, peso medio $48.56 \pm 10.82 \mathrm{~kg}$ y talla media $159.45 \pm 7.38 \mathrm{~cm}$ ). Las atletas se dividieron en dos grupos de acuerdo con su nivel competitivo (élite, $n=4$ y no élite, $n=13$ ). Las atletas élite fueron participantes en la primera categoría individual en el año 2017 (categoría que incluye las 18 mejores gimnastas de España), por lo que suponen el 22.22\% del total de gimnastas de ese nivel. Entrenaban 8 sesiones de entre 3 y 4 horas por semana. Las atletas no élite participaron en el "Campeonato Nacional Base individual" en el año 2017 (campeonato con unas 150 participantes por categoría, por lo que suponen un $9.3 \%$ del total de gimnastas de su categoría). Entrenaban 4 sesiones de entre 3 y 4 horas por semana. Las características descriptivas de las gimnastas de cada grupo se encuentran en la Tabla 1. Todas las participantes y sus familias fueron informadas con detalle sobre el estudio de manera previa a la realización de los tests. Aportaron un consentimiento informado firmado por su padre/ madre o tutor autorizando su participación en el estudio, aprobado por el Comité de Ética de la Universidad de Alicante.
Tabla 1. Características descriptivas de las participantes. Valores medios y desviación estándar.

\begin{tabular}{ccc}
\hline Variable & No Élite $(\mathrm{n}=14)$ & Élite $(\mathrm{n}=4)$ \\
\hline Edad (años) & $14.48 \pm 1.21$ & $14.78 \pm 1.21$ \\
Estatura $(\mathrm{cm})$ & $159.21 \pm 6.80$ & $160.3 \pm 10.35$ \\
Masa $(\mathrm{kg})$ & $49.71 \pm 11.78$ & $44.53 \pm 5.93$ \\
\hline
\end{tabular}

Instrumentos y procedimiento

Los tests se realizaron en dos sesiones, separadas por 24 horas. Todas las gimnastas realizaron las pruebas en las mismas condiciones, tanto previas como durante su desarrollo: sin haber entrenado el día anterior ni el mismo día, y a la misma hora de la tarde. En la primera sesión se evaluaron características antropométricas, capacidad de salto y flexibilidad. En la segunda se realizó un test de campo consistente en la realización de un ejercicio competitivo en el que se registró la frecuencia cardíaca pre, durante y post ejercicio, y se midieron los niveles de lactato al finalizar el mismo. El protocolo de todos los tests era conocido por las gimnastas y había sido practicado la semana previa para evitar la influencia del aprendizaje en los resultados. Antes de la primera sesión se realizó un calentamiento de una duración de 20-25 min consistente en:

- Movilidad articular estática de extremidades inferiores, superiores y tronco (2 min).

- Carrera combinada con movilidad articular dinámica (5 min): circunducciones de brazos, skipping, talones al glúteo y carrera lateral.

- Saltos con cuerda: $15 \mathrm{x}$ hacia delante, $15 \mathrm{x}$ hacia atrás.

- En una barra de ballet: lanzamientos de pierna (battements) hacia delante, laterales y hacia atrás. 8 repeticiones con cada pierna aguantando en la última con y sin ayuda de la mano. Repetir todo dos veces, primero elevando la pierna hasta $90^{\circ} \mathrm{y}$ después al máximo.

- Saltos con cuerda: 15 x cruzados y 15 x dobles.

El segundo día se realizó el mismo calentamiento, pero tras él se dejaron 15 minutos extra para que cada gimnasta repasara de manera autónoma las dificultades de aparato y otros aspectos del ejercicio competitivo que iban a realizar en el test.

\section{Antropometría}

Se realizaron una serie de medidas antropométricas, en línea con los estudios realizados por Douda et al. (2008), Di Cagno et al. (2008a, 2009) y Donti et al., (2016). Se siguió el procedimiento estandarizado 
de la Sociedad Internacional para el Avance de la Cineantropometría (2005). Se registraron la estatura, masa corporal, cuatro diámetros (biacromial, bicrestal, fémur y muñeca), seis perímetros (brazo relajado, brazo contraído, muslo medio, muslo máximo de ambas piernas y pierna) y ocho pliegues cutáneos (bicipital, tricipital, subescapular, supraespinal, abdominal, muslo y pierna).

La estatura fue medida sin zapatos con un tallímetro de $0.1 \mathrm{~cm}$ de sensibilidad (Mediprem) y la masa corporal con una báscula digital de $0.1 \mathrm{~kg}$ de sensibilidad (Soehnle, modelo Style Sense Compact 100). Los diámetros fueron medidos con un paquímetro de $0,1 \mathrm{~cm}$ de sensibilidad (Cescorf, modelo Innovare) y los perímetros con una cinta métrica de $0,1 \mathrm{~cm}$ de sensibilidad. Los pliegues se tomaron con un calibre Cescorf modelo Innovare 2, de $1 \mathrm{~mm}$ de sensibilidad. Todas las medidas fueron tomadas dos veces por el mismo evaluador. En el caso de encontrar en alguna de ellas una diferencia superior a un $10 \%$ se tomó una tercera. El dato de la medida de cada pliegue se corresponde con la media de las medidas realizadas para dicho pliegue.

Los datos antropométricos se emplearon para su comparación entre ambos grupos y el cálculo del porcentaje de grasa corporal, determinado mediante la fórmula de Faulkner (1968), recomendado por Alvero et al. (2010) para el tipo de muestra analizado.

\section{Capacidad de salto}

Se evaluó la capacidad de salto como indicador de la potencia de piernas, siguiendo los estudios de Di Cagno et al. (2008a,2009) y Donti et al., (2016). Se siguió la metodología descrita por Bosco (1992). Los sujetos realizaron tres variaciones del salto vertical en el siguiente orden: salto sin contramovimiento (SJ), salto con contramovimiento (CMJ) y salto con caída desde un cajón de 0,3 m (DJ). El propósito de este último era el cálculo del índice de reactividad, por lo que no se realizó protocolo de alturas incrementales. Se realizaron tres intentos de cada prueba con un tiempo mínimo de descanso de 45" entre cada uno, y fue tomado para su análisis el máximo valor. Se realizó una serie de prueba antes de comenzar a registrar los resultados.

Los saltos fueron grabados y analizados mediante la aplicación Myjump instalada en el dispositivo Ipad Mini 4, a una frecuencia de captura de $30 \mathrm{fps}$. Esta aplicación fue creada y validada de manera reciente y se considera un método fiable y válido para la medida de la altura de salto y el cálculo del índice de reactividad (Balsalobre-Fernández, Glaister, \& Lockey, 2015; Stanton, Wintour, \& Kean, 2017).
También se realizó un Hopping test (HT), consistente en una serie de siete saltos continuos con brazos libres, con contramovimiento de poca amplitud y un breve tiempo de contacto con el suelo. Se evaluó el tiempo de realización del mismo (Di Cagno et al., 2008a; 2009). La duración del test fue grabada con el dispositivo móvil Iphone 8 a una frecuencia de captura de $60 \mathrm{fps}$, y posteriormente analizada con el software Kinovea.

\section{Flexibilidad}

Esta capacidad se evaluó mediante una batería de test detallados a continuación. En todos los casos se realizaron dos medidas y se seleccionó la mejor para su posterior análisis.

Sit and reach (SR en Figura 1): se empleó para evaluar la flexibilidad de la musculatura isquiotibial y la movilidad de la articulación de la cadera de manera general (Donti et al., 2016) y (Douda et al., 2008). Para la realización de esta prueba se siguió el protocolo establecido por Cramer y Coburn (2004). Se fijó en el suelo una cinta métrica de $0,1 \mathrm{~cm}$ de sensibilidad, realizando una marca a partir de la cual se medirá. A continuación, la gimnasta se coloca sentada en el suelo, con las piernas a una distancia de $0,25 \mathrm{~m}$ entre ellas y los pies a la altura de la marca, dejando la cinta métrica en el medio. Desde esa posición, realizará una flexión progresiva del tronco con los brazos hacia delante, tratando de alcanzar el punto más alejado de los pies posible. Deberá ser capaz de aguantar esa posición durante 2".

Elevación frontal de la pierna (EF en Figura 1): este test se empleó para evaluar la flexibilidad y rango de movimiento de la musculatura isquiotibial y la articulación de la cadera de una manera más específica (Donti et al., 2016; Donti, Tsolakis, \& Bogdanis, 2014). La gimnasta debe colocarse tumbada en el suelo en posición supina sin despegar el dorso del suelo en ningún momento del test. La pierna que no se eleva será sujetada por un asistente para evitar la flexión de la rodilla. La gimnasta elevará la otra pierna con ayuda de los brazos hasta el máximo punto posible, con la rodilla completamente extendida. En esta posición se tomó una imagen con la cámara del teléfono móvil Iphone 8, para posteriormente calcular el rango de movimiento en grados mediante el software informático Kinovea. Para este cálculo, una de las líneas del medidor se clocó en la línea media lateral de la pelvis, mientras que la otra se posicionó en la línea media lateral del fémur, conectando el trocánter mayor de la pelvis con el epicóndilo lateral de la rodilla.

Elevación lateral de la pierna (EL en Figura 1): este test fue propuesto por Douda et al. (2008) para evaluar 

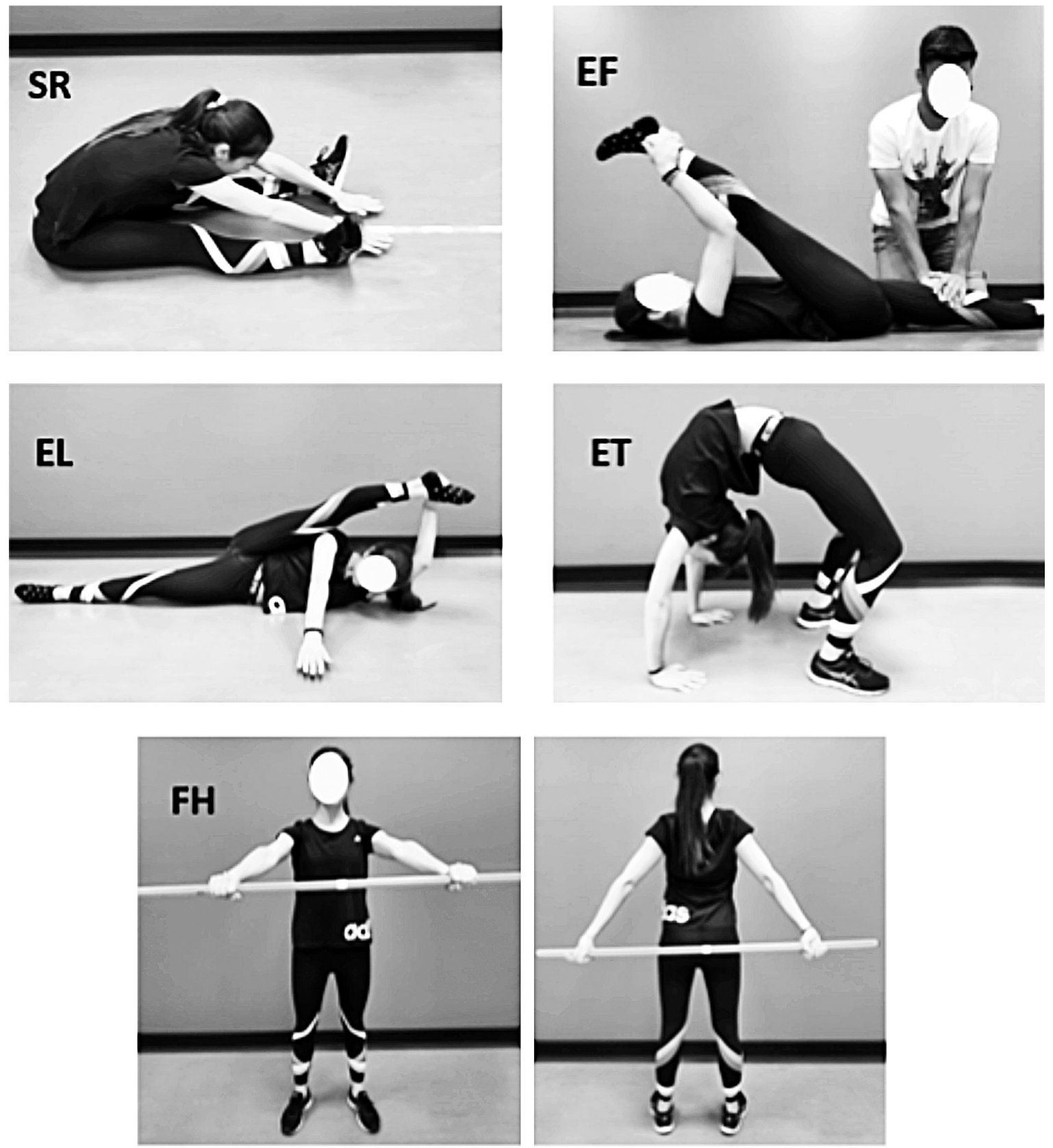

Figura 1. Test de evaluación de la flexibilidad.

la movilidad de la cadera. La gimnasta debe tumbarse en el suelo de manera lateral, elevando una pierna lo máximo posible con ayuda de las manos ("battement a la seconde") sin que se despegue ninguna parte de la pierna ni de la cadera de la pierna que se encuentra en el suelo. En esa posición, con una cinta métrica de precisión 0,1 cm se mide la distancia entre el maléolo lateral de la pierna elevada y el suelo.

Test de Extensión de tronco (ET en Figura 1): este test, realizado por Donti et al. (2016) para una muestra de las mismas características, consiste en la medida de la distancia entre muñecas y talones en la posición de puente (Rutkauskaite \& Skarbalius, 2011). Para minimizar el error de la medida, ya que la altura de la gimnasta puede afectar a esta distancia, se ha empleado la fórmula diseñada por Donti et al. (2016) con ese fin, en la que se mide la estatura de la gimnasta con los brazos levantados (hasta la muñeca), para después restarle a ese valor el obtenido en el test, dividiendo esta cifra entre la estatura con los brazos extendidos.

Flexibilidad de hombros (FH en Figura 1): se evaluó mediante un test diseñado por Douda et al. (2008). La 
gimnasta debe agarrar una barra de madera con una marca de 0,1 cm en su parte media con las manos en pronación, y levantarla por encima de la cabeza hasta pasarla detrás de la espalda, manteniendo el agarre sin cambios y los codos extendidos. El test se deberá repetir, juntando las manos entre sí de manera progresiva en cada repetición, hasta que la gimnasta no sea capaz de completar el movimiento. La distancia entre las manos en ese momento será el valor registrado.

\section{Lactato y frecuencia cardíaca en entorno} de competición

Estas medidas se llevaron a cabo mediante la realización de un test de campo específico basado en el implementado por Guidetti et al. (2000). Consiste en la realización por parte de cada gimnasta de su ejercicio competitivo, registrando la frecuencia cardíaca desde los $5 \mathrm{~min}$ anteriores al test (en reposo), durante y después del mismo hasta los 10 min de recuperación (también en reposo). Para el registro de esta variable, se emplearon monitores portátiles de Frecuencia Cardíaca (Sistema Polar Team 1, Polar Electro).

En cuanto a la determinación de los niveles de lactato en sangre, se empleó el lactacidómetro portátil Lactate Scout+. La toma de muestras se llevó a cabo inmediatamente después de la recolección de sangre capilar de la yema del dedo. Las medidas se realizaron justo al finalizar la prueba, a los 3 y a los $10 \mathrm{~min}$ de recuperación.

Los ejercicios competitivos realizados por las gimnastas cumplían con los requerimientos técnicos exigidos por el código de puntuación para sus respectivos niveles. Las gimnastas del nivel inferior contaban con siete elementos de dificultad corporal de los cuales en todos los casos tres eran saltos, dos eran equilibrios y tres eran giros. Todas ellas contaban con dos series de pasos rítmicos. Las gimnastas de nivel élite por su parte contaban con nueve dificultades corporales en proporción variable de cada uno de los grupos en función de cada gimnasta, así como una sola serie de pasos rítmicos.

Cada gimnasta ejecutó el ejercicio con el implemento seleccionado por sus respectivas entrenadoras. No obstante, se excluyó la cuerda, ya que según Guidetti et al. (2000) se reportan valores de frecuencia cardíaca y lactato similares para todos los implementos excepto la cuerda, que se considera el más extenuante.

\section{Análisis de datos}

Se han calculado las variables estadísticas descriptivas (media, desviación estándar y porcentaje de di- ferencia entre grupos) para todos los datos obtenidos mediante el software Excel versión 2013.

En cuanto al análisis estadístico para calcular las diferencias significativas entre grupos ha sido realizado con el programa IBM SPSS Statistics versión 24.0. Se ha realizado la prueba de Levene para asegurar la normalidad de la muestra. Una vez asegurada, se asumen los valores de " $p$ " con varianzas iguales para analizar las diferencias significativas. Los niveles de significación de " $p$ ” fueron ${ }^{*} p<0.05 ;{ }^{* *} p<0.01$. Para los valores de $p>0.01$ se considera que no existen diferencias significativas.

\section{Resultados}

Los resultados de las medidas antropométricas, junto con los porcentajes de diferencia entre ambos grupos, se presentan en la Tabla 2.

Los resultados de los tests físicos realizados, tanto de salto como de flexibilidad, se encuentran en la Tabla 3. Las gimnastas élite reportan valores mayores en todos los test de flexibilidad. En cuanto a los tests de salto, observamos mayores valores en el grupo élite en CMJ y SJ, y sobre todo en el índice de reactividad en el DJ. En cuanto al tiempo de contacto en el hopping test, el grupo no élite obtuvo un mejor resultado, pero la diferencia es escasa.

Por último, los datos obtenidos en el test de campo respecto a los parámetros fisiológicos de frecuencia cardíaca y lactato se presentan en las Figuras 1, 2 y 3 . No se encontraron diferencias significativas entre grupos en ninguno de los momentos registrados de la frecuencia cardíaca (Reposo $p=0.923$; inicial $p=$ 0.633 ; media $p=0.416$; pico $p=0.458$; final $p=0.277$; recuperación 3' $p=0.522$; recuperación 10' $p=0.297$ ) Los valores de lactato en sangre son más dispares, encontrando un valor superior en el grupo élite justo al finalizar la prueba. En cuanto a los valores tomados a los 3' y 10' son menores en el grupo élite en ambos casos. No obstante, tampoco se encontraron diferencias significativas en ninguno de los momentos registrados de lactato (Final $p=0.126$; recuperación 3' $p=0.733$; recuperación 10’ $p=0.297$ ).

\section{Discusión}

El objetivo del presente trabajo era comparar dos grupos de gimnastas españolas de diferentes niveles y establecer las diferencias existentes entre ambos, relacionándolos con los factores de rendimiento antropométricos, físicos y fisiológicos estudiados en la literatura existente. 
Tabla 2. Resultados de las medidas antropométricas: Perímetros, pliegues cutáneos y diámetros. Sumatorio de pliegues cutáneos y porcentaje de grasa.

\begin{tabular}{|c|c|c|c|c|}
\hline Variables antropométricas & No élite $(n=14)$ & Élite $(n=4)$ & \%Diferencia & Diferencias significativas ( $p)$ \\
\hline Perímetro brazo relajado $(\mathrm{cm})$ & $24.46 \pm 2.71$ & $21.88 \pm 1.73$ & -10.58 & 0.093 \\
\hline Perímetro brazo contraído $(\mathrm{cm})$ & $25.67 \pm 2.62$ & $23.70 \pm 1.60$ & -7.68 & 0.177 \\
\hline Perímetro muslo medio $(\mathrm{cm})$ & $48.08 \pm 4.17$ & $42.15 \pm 2.36$ & -12.33 & $0.016^{*}$ \\
\hline Perímetro pierna (cm) & $33.96 \pm 2.80$ & $31.53 \pm 1.91$ & -71.6 & 0.126 \\
\hline Pliegue bicipital (mm) & $3.87 \pm 2.32$ & $1.25 \pm 1.19$ & -67.71 & $0.047^{*}$ \\
\hline Pliegue tricipital (mm) & $10.07 \pm 5.48$ & $5.25 \pm 2.53$ & -47.87 & 0.112 \\
\hline Pliegue subescapular (mm) & $7.48 \pm 3.84$ & $4.25 \pm 1.32$ & -43.17 & 0.124 \\
\hline Pliegue abdominal (mm) & $8.43 \pm 4.37$ & $3.63 \pm 2.29$ & -56.99 & 0.053 \\
\hline Pliegue supraespinal (mm) & $10.29 \pm 4.28$ & $5.75 \pm 3.23$ & -44.10 & 0.069 \\
\hline Pliegue iliocrestal (mm) & $5.11 \pm 2.63$ & $2.50 \pm 1.58$ & -51.05 & 0.081 \\
\hline Pliegue muslo (mm) & $15.50 \pm 6.40$ & $11.25 \pm 5.97$ & -27.42 & 0.253 \\
\hline Pliegue pierna (mm) & $9.14 \pm 5.80$ & $4.75 \pm 3.71$ & -48.05 & 0.176 \\
\hline Diámetro biacromial (cm) & $35.99 \pm 1.93$ & $36.75 \pm 1.19$ & -2.10 & 0.473 \\
\hline Diámetro bicrestal (cm) & $21.98 \pm 0.92$ & $20.63 \pm 0.75$ & -6.16 & $0.016^{*}$ \\
\hline Diámetro del fémur (cm) & $9.07 \pm 0.53$ & $8.40 \pm 0.22$ & -7.40 & $0.028^{*}$ \\
\hline Diámetro de la muñeca $(\mathrm{cm})$ & $4.84 \pm 0.29$ & $4.70 \pm 0.08$ & -2.81 & 0.143 \\
\hline Sumatorio de pliegues (mm) & $69.89 \pm 31.48$ & $38.63 \pm 20.95$ & -44.73 & 0.083 \\
\hline$\%$ Grasa (Faulkner) & $15.62 \pm 3.58$ & $11.92 \pm 1.95$ & -23.71 & 0.068 \\
\hline
\end{tabular}

Diferencias significativas ${ }^{*} \mathrm{p}<0.05 ;{ }^{*} \mathrm{p}<0.01$

Tabla 3. Resultados de los tests de salto y flexibilidad.

\begin{tabular}{|c|c|c|c|c|}
\hline Variables físicas & No élite $(n=14)$ & Élite $(n=4)$ & $\%$ Diferencia & Diferencias significativas ( $p)$ \\
\hline \multicolumn{5}{|l|}{ Saltos verticales } \\
\hline $\mathrm{CMJ}(\mathrm{cm})$ & $24.60 \pm 2,22$ & $27.21 \pm 1.23$ & 10.58 & $0.041 *$ \\
\hline $\mathrm{SJ}(\mathrm{cm})$ & $24.00 \pm 2,68$ & $26.73 \pm 1.36$ & 11.37 & 0.071 \\
\hline DJ (índice de reactividad) & $1.07 \pm 0,25$ & $1.66 \pm 0.45$ & 55.22 & $0.003 * *$ \\
\hline Hop test, tiempo de contacto (s) & $1.34 \pm 0,12$ & $1.29 \pm 0.06$ & -3.73 & 0.428 \\
\hline \multicolumn{5}{|l|}{ Tests de flexibilidad } \\
\hline Sit and reach $(\mathrm{cm})$ & $69.43 \pm 2,41$ & $72.63 \pm 3.59$ & 4.60 & 0.051 \\
\hline Test flexibilidad de hombro $(\mathrm{cm})$ & $41.86 \pm 11,11$ & $22.38 \pm 20.38$ & -46.65 & $0.02 *$ \\
\hline Elevación lateral pierna derecha $(\mathrm{cm})$ & $33.40 \pm 6,40$ & $18.38 \pm 5.85$ & -44.99 & $0.001 * *$ \\
\hline Elevación lateral pierna izquierda $(\mathrm{cm})$ & $40.40 \pm 6,36$ & $24.18 \pm 5.37$ & -40.16 & $0.003 * *$ \\
\hline Elevación frontal de pierna derecha estirada $\left(^{\circ}\right)$ & $134.00 \pm 9,72$ & $160.00 \pm 13.09$ & 19.40 & $0.021 *$ \\
\hline Elevación frontal de pierna izquierda estirada $\left(^{\circ}\right)$ & $121.07 \pm 12,44$ & $145.25 \pm 12.50$ & 19.97 & $0.02 *$ \\
\hline Puente (coeficiente) & $0.88 \pm 0,08$ & $0.88 \pm 0.03$ & 0.06 & 0.993 \\
\hline
\end{tabular}

Diferencias significativas ${ }^{*} p<0.05 ;{ }^{*} p<0.01$.

En líneas generales, los resultados han coincidido con los reportados en la literatura publicada hasta el momento, encontrado grandes diferencias entre grupos en todas las variables evaluadas. En cuanto a las diferencias estadísticas (significativas), han sido muy escasas, debido a las características de la muestra, muy reducida en uno de los grupos (élite, $n=4$ ) y heterogénea (las desviaciones estándar en muchos de los casos son elevadas). Por otro lado, se han hallado también ciertas diferencias respecto a la literatura, que se detallarán en líneas posteriores.

Según numerosos autores, la antropometría es uno de los principales factores de rendimiento en la gimnasia rítmica, caracterizándose las gimnastas por una baja masa corporal y unas extremidades largas y delgadas (Douda et al., 2008; Malina et al., 2013; Purenović-Ivanović \& Popović, 2014).

Di Cagno et al. (2008a) afirma que a medida que el nivel de la muestra se eleva se reducen las diferencias interindividuales. En contraposición, se han registrado desviaciones estándar elevadas en muchas de las medidas realizadas no solo con las gimnastas de menor nivel sino también con el grupo élite. Esto podría deberse a las discrepancias entre las muestras del estudio citado y el presente trabajo, pudiendo señalar dos factores: en primer lugar, el nivel: las gimnastas élite evaluadas por Di Cagno et al. (2008a) son medallistas olímpicas, mientras que la muestra élite del presente estudio está formada por medallistas en campeonatos nacionales y torneos internacionales. También podría 


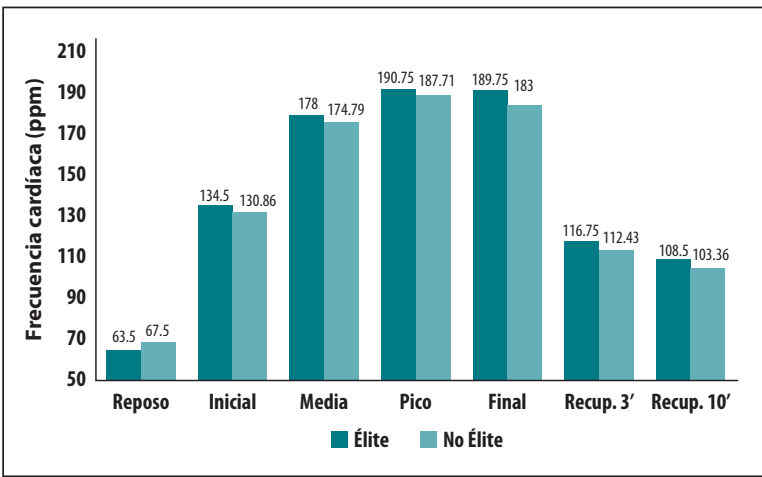

Figura 2. Resultados de los valores de Frecuencia Cardíaca obtenidos en el test de campo.

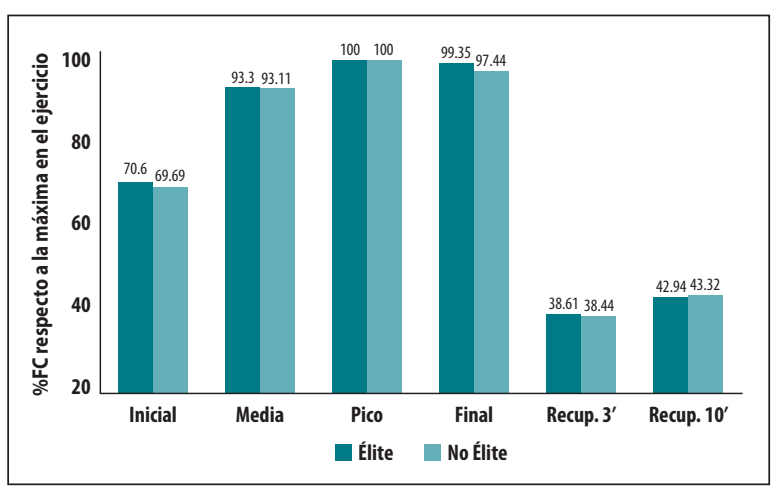

Figura 3. Valores de Frecuencia cardíaca obtenidos en el test de campo expresados en porcentaje en relación a la máxima obtenida durante el test.

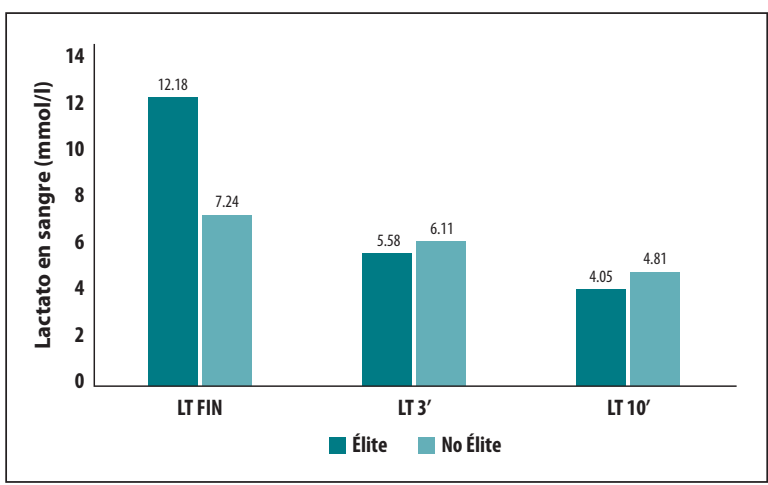

Figura 4. Valores de concentración de lactato en sangre post-ejercicio obtenidos en el test de campo.

deberse a la modalidad de las deportistas; aquellas evaluadas en el artículo citado pertenecen a la modalidad de conjunto. Generalmente, a la hora de llevar a cabo el proceso de selección de talentos para un conjunto se tiene en cuenta que todas sus integrantes sean de dimensiones antropométricas similares, lo que reduce al mínimo las diferencias interindividuales, algo que no sucede en la modalidad individual.

En cuanto a la masa grasa, el grupo de gimnastas no élite presenta de media un $23.72 \%$ más que las gimnastas élite, así como un sumatorio de 8 pliegues un $44 \%$ mayor. A la hora de comparar los resultados con la literatura existente, podemos ver cómo los datos obtenidos en cuanto a pliegues cutáneos y diámetros se asemejan a los obtenidos en otros estudios como Purenović-Ivanović \& Popović (2014) para el grupo élite, mientras que difieren ligeramente de los obtenidos en otros estudios como Douda et al. (2008), quienes obtuvieron menores resultados en los perímetros evaluados, lo que se puede explicar debido a la edad media de la muestra evaluada que, a pesar de encontrarse dentro de la misma categoría de edad, era un año menor. En cuanto a los valores de porcentaje de grasa encontramos ciertas diferencias en su comparación, que pueden derivarse del uso de diferentes fórmulas para su cálculo (Di Cagno et al. 2008a; Douda et al. 2008). En cuanto al sumatorio de pliegues, no ha sido posible su comparación con otros estudios, ya que no se han reportado trabajos hasta el momento que midan todos los pliegues que se han tomado en este caso.

No obstante, al igual que en los estudios previos, observamos cómo las gimnastas de mayor nivel poseen un menor porcentaje de grasa y son más ligeras y con extremidades más largas. Esto relaciona de manera clara la antropometría con el rendimiento, ya que implicará una mayor facilidad para ejecutar los movimientos requeridos (Douda et al., 2008, Sinning, 1978). Además, nos encontramos en un deporte en el que una apariencia estética delgada y estilizada se asocia a una mejor predisposición a la hora de puntuar por parte del jurado (Hume et al., 1993; Malina et al., 2013).

Según Di Cagno et al. (2009), la antropometría influye de manera directa en otros factores de rendimiento en este deporte como la capacidad de salto, viéndose favorecida por una mayor estatura, longitud de extremidades y una menor masa corporal y porcentaje de grasa. A pesar de que es importante tener en cuenta las limitaciones debidas a los diferentes evaluadores y material empleado, esta afirmación respaldaría los resultados obtenidos en los test de salto, ya que las gimnastas del grupo élite han obtenido valores superiores en la altura de vuelo en el SJ (+11.33\%) y CMJ (+10.58\%), así como en el índice de reactividad en el DJ (+55.22\%). Observamos que la mayor diferencia entre grupos se encuentra en este último parámetro, encontrando una notable diferencia significativa $(p=0,03)$, hecho que sustenta la literatura científica, ya que, según Di Cagno et al. (2008a) la reactividad de las extremidades inferiores es uno de los indicadores más fiables para la detección de talentos en gimnasia rítmica, y el DJ es un test validado para medir este parámetro (Arampatzis, Brüggemann, \& Klapsing, 2001; Lockie, Schultz, Callaghan, Jeffriess, \& Luczo, 2014; Stanton et al., 2017) . Por otra parte, al igual que en Di Cagno et al., (2008a), 
las diferencias encontradas en el tiempo de contacto del Hopping Test son muy reducidas, siendo el grupo no élite tan solo un $3.73 \%$ superior. Esto podría explicarse de acuerdo a las exigencias técnicas del deporte, ya que la altura del salto influye en la puntuación de manera destacable ( Di Cagno et al., 2009) mientras que no se encuentran afirmaciones acerca de la influencia de la velocidad de los saltos en la puntuación total.

La flexibilidad es otro de los principales factores de rendimiento en este deporte (Donti et al., 2016; Douda et al., 2008). Según los resultados obtenidos en los tests evaluados, las gimnastas del grupo élite son superiores en todos ellos, encontrando diferencias significativas en los tests de flexibilidad de hombro, elevación lateral y frontal de la pierna. Las mayores diferencias entre grupos se encuentran en los dos primeros (46.55\% y $44.99 \%$ respectivamente), aunque cabe mencionar que en los resultados del test de hombro existe una desviación estándar muy elevada (20,38 $\mathrm{cm}$ ) debido a las grandes diferencias interindividuales registradas. Las menores diferencias entre grupos se encuentran en los tests de extensión de espalda y sit and reach $(0.06 \%$ y $4.6 \%$, respectivamente). Estos resultados respaldan la literatura existente, ya que según Donti et al. (2016), la extensión lateral de la pierna es el mejor predictor de la puntuación técnica en gimnasia rítmica en todos los niveles, y uno de los factores de rendimiento que permiten discriminar a las atletas según su nivel. Además, señala que el rango de movimiento del hombro es de gran importancia a la hora de manipular los implementos a una distancia óptima del cuerpo y de realizar los elementos pre-acrobáticos con apoyo de manos en el suelo. De acuerdo con Donti et al. (2016), rangos de movimiento elevados en hombro y cadera protegen a las gimnastas de las cargas excesivas en la columna vertebral durante la realización de posturas "extremas".

Los resultados existentes en la literatura son muy similares a los obtenidos en el presente estudio en cuanto a la flexibilidad de hombro en el grupo élite, y difieren en todos los demás aspectos en ambos grupos, obteniendo mejores resultados en todos los casos los sujetos participantes en los estudios ya realizados (Donti et al., 2016; Douda et al., 2008). Esto podría deberse a que actualmente el nivel de gimnasia individual en España es inferior al de otros países como Grecia, del que proceden los sujetos de los artículos hallados, hecho que se puede constatar con los resultados obtenidos por ambos países en competiciones internacionales, como el Campeonato de Europa de 2017, en el que las gimnastas griegas quedaron 7, 14 y 15 puestos por delante respectivamente en tres de los cuatro aparatos que se realizaron en la competición.
Cabe destacar que, respecto a los factores de rendimiento fisiológicos, la literatura hallada es escasa y poco específica. Los artículos publicados suelen emplear tests incrementales en cicloergómetro para determinar parámetros como la frecuencia cardíaca máxima, el lactato o el consumo máximo de oxígeno (Baldari \& Guidetti, 2001; Di Cagno et al., 2008; Douda et al., 2008). Estos tests someten a los sujetos a condiciones muy diferentes a las propias de la competición en el deporte, puesto que en ellos el esfuerzo es continuo e incremental durante aproximadamente 15 minutos, realizando un esfuerzo cíclico que involucra al tren inferior principalmente. Por el contrario, un ejercicio de Gimnasia Rítmica tiene una duración de 90 segundos en los que la gimnasta combina ininterrumpidamente diversos elementos de muy alta intensidad que involucran todos los grupos musculares, como saltos o carreras, con un manejo continuo de los implementos y ciertos momentos algo menos intensos. Tan solo un artículo encontrado evalúa a su muestra a través de un test de campo en el que se registran los parámetros fisiológicos de frecuencia cardíaca, lactato y consumo de oxígeno durante la ejecución de un ejercicio competitivo (Guidetti et al., 2000).

Los valores de frecuencia cardíaca media y máxima registrados durante el ejercicio de las gimnastas del grupo élite es muy similar a la reportada para gimnastas del mismo nivel por Guidetti et al. (2000). Las diferencias de estos valores con los obtenidos en el grupo no élite son muy pequeñas, lo que nos hace pensar que, a pesar de no disponer de literatura al respecto para comparar los niveles inferiores, no existen diferencias significativas entre ambos grupos. Esto podría explicarse de la siguiente manera: todas las gimnastas realizan los ejercicios competitivos de acuerdo al máximo de sus posibilidades y capacidades físicas y técnicas, por lo que la carga interna que les supone es muy elevada en todos los casos independientemente del nivel competitivo (Douda et al., 2008). Podemos observar también que en ambos grupos la frecuencia cardíaca de inicio del ejercicio es bastante superior a la de reposo. Esto podría deberse tanto al estrés emocional de la competición como a las posiciones isométricas de inicio (Alexander, Boreskie \& Law, 1987).

Según Guidetti et al. (2000), los valores medios de lactato al finalizar un ejercicio se encuentran en torno a los $4.0 \pm 0.4 \mathrm{mmol} / \mathrm{l}$ en gimnastas élite en todos los implementos, no existiendo datos en la literatura sobre las gimnastas de niveles inferiores. Los resultados obtenidos en el presente estudio muestran valores muy superiores. No obstante, no es posible realizar una comparación real entre ambos valores ya que en el año 2000 el código de puntuación en base al cual se 
construían los ejercicios era muy diferente al actual, por lo que los requerimientos físicos y por tanto energéticos eran diferentes también.

Si nos ceñimos a los resultados obtenidos, en ambos grupos encontramos valores por encima de los 5 mmol/l, lo que, según Bourdon (2013) indicaría que los ejercicios competitivos se sitúan entre el umbral anaeróbico y la potencia anaeróbica máxima. Esto se encuentra respaldado también por los valores relativos de frecuencia cardíaca durante el ejercicio. En ambos grupos se encuentra en un $93 \%$ sobre la máxima alcanzada. Estos resultados siguen la línea de Guidetti et al. (2000), que establece el consumo máximo de oxígeno, asociado al 95-100\% de la frecuencia cardíaca durante el ejercicio, como uno de los principales determinantes del rendimiento en gimnasia rítmica. Podemos encontrar valores muy superiores de lactato al finalizar el ejercicio en el grupo de las gimnastas élite ( $86 \%$ mayor). Esto podría deberse a la diferencia en la velocidad de los movimientos en los ejercicios, siendo esta mucho más elevada en niveles superiores, donde las gimnastas cuentan con las destrezas corporales y de aparato suficientes como para realizar más acciones en un mismo tiempo que las gimnastas de menor nivel, las cuales necesitan un mayor tiempo de preparación para cada acción dentro del ejercicio. Otra posible causa podría ser la difícil estabilización de los niveles de lactato en ejercicios de una duración inferior a 3 minutos (Bourdon, 2013), siendo los ejercicios de gimnasia de 90 segundos. A pesar de mostrar valores de finalización más elevados, observamos que la recuperación en el grupo élite es mucho mayor tanto a los 3 como a los 10 minutos post-ejercicio. Esto podría deberse a un mayor nivel de condición física y una mayor necesidad de reciclaje del lactato. Estas gimnastas se encuentran más acostumbradas a entrenamientos más largos, con un mayor volumen de repeticiones de los ejercicios, por lo que se intuye que su capacidad para reciclar el lactato es superior a las gimnastas de menor nivel, acostumbradas a un mayor tiempo de descanso entre repeticiones.

\section{Limitaciones}

Como principal limitación del presente estudio podríamos señalar la falta de investigaciones recientes publicadas en esta área. Esto dificulta las comparaciones con resultados previos, ya que el código de puntuación por el que se rigen los ejercicios competitivos se va modificando, lo que modifica también las exigencias físicas y fisiológicas de los mismos. Actualmente no existe ningún estudio elaborado con el código vigente, con lo que las comparaciones establecidas en cuanto a los valores de los parámetros analizados deben tomarse con cautela. Otra limitación reside en la comparación de diferentes ejercicios en los que el número y tipo de elementos ejecutados, aunque similar, no era el mismo (algunas gimnastas sobre todo élite ejecutaban más saltos; otras, más giros, etc.), lo que provocaría que el esfuerzo demandado para cada una de ellas no fuera exactamente igual.

Por otra parte, a la hora de establecer las relaciones entre las variables medidas y los diferentes niveles no se han tenido en cuenta las puntuaciones obtenidas en competición por cada una de las gimnastas evaluadas para así poder correlacionar esta variable con el rendimiento (Di Cagno et al., 2014), ya que no se ha podido disponer de las mismas. En futuros estudios sería interesante añadir esta variable para obtener resultados más concluyentes.

En cuanto al análisis estadístico, el tamaño de la muestra también supone una limitación, ya que el número de participantes del grupo élite, a pesar de suponer un porcentaje elevado sobre el total de la muestra, es muy escaso $(n=4)$. Esto, unido a la disparidad entre las participantes de cada grupo, hace que, a pesar de encontrar grandes diferencias porcentuales entre uno y otro, las diferencias significativas sean escasas.

\section{Conclusiones}

En conclusión, teniendo en cuenta las limitaciones del trabajo, los resultados han mostrado que los valores antropométricos, la capacidad de salto, la flexibilidad y la capacidad de reciclaje del lactato serían los principales factores de rendimiento a evaluar para diferenciar a las gimnastas en función de su nivel. Un menor porcentaje de grasa y menor masa corporal llevarán a una mayor capacidad de salto, una mayor flexibilidad permitirá ejecutar dificultades de mayor valor, y una mayor capacidad de reciclar el lactato permitirá una mejor recuperación entre ejercicios para afrontarlos con más garantías. Por otra parte, es necesario tener en cuenta que el rendimiento en Gimnasia Rítmica también se verá muy determinado por la destreza técnica de manejo de los implementos, y la coordinación necesaria para ejecutar las dificultades de aparato de mayor dificultad posible de manera correcta. No obstante, los factores medibles evaluados en el presente estudio podrían utilizarse como identificadores del rendimiento deportivo en gimnasia, ayudando a la elaboración de los programas de entrenamiento y detección de talentos por parte de los entrenadores. 


\section{BIBLIOGRAFÍA}

Alexander M.J.L, Boreskie S.R \& Law S (1978). Heart rate response time and motion analysis of rhythmic sportive gymnasts. Journal of $\mathrm{Hu}$ man Moviment studies; 13: 473-489

Alvero, J.R., Cabanillas, M.D., Herrero, A., Martínez, L., Moreno, C., Porta, J., ... Sirvent, J.E. (2010). Protocolo de valoración de la composición corporal para el reconocimiento médico deportivo. Documento de consenso del Grupo Español de Cineantropometría (GREC) de la Federación Española de Medicina del Deporte (FEMEDE). Archivos de medicina del deporte, 27(139), 330-334. Recuperado de: http://femede.es/documentos/ConsensoCine131.pdf

Arampatzis, A., Brüggemann, G. P., \& Klapsing, G. M. (2001). Leg stiffness and mechanical energetic processes during jumping on a sprung surface. Medicine and Science in Sports and Exercise, 33(6), 923-31. Recuperado de: http://www.ncbi.nlm.nih.gov/pubmed/11404657

Baldari, C., \& Guidetti, L. (2001). VO2max, ventilatory and anaerobic thresholds in rhythmic gymnasts and young female dancers. The Journal of Sports Medicine and Physical Fitness, 41(2), 177-82. Recuperado de: http://www.ncbi.nlm.nih.gov/pubmed/11447359

Balsalobre-Fernández, C., Glaister, M., \& Lockey, R. A. (2015). The validity and reliability of an iPhone app for measuring vertical jump performance. Journal of Sports Sciences, 33(15), 1574-1579. doi:10.1080 /02640414.2014.996184.

Benardot, D., \& Czerwinski, C. (1991). Selected body composition and growth measures of junior elite gymnasts. Journal of the American Dietetic Association, 91(1), 29-33. Recuperado de: http://www.ncbi.nlm. nih.gov/pubmed/1869756

Bosco C. (1992). Elasticitá muscolare e Forza esplosiva nelle attivitá físico-sportive. Roma: Societá Stampa Sportiva.

Bourdon, P. (2013). Blood lactate thresholds: Concepts and Applications. En R.K Tanner \& C.J Gore (Ed.). Physiological tests for elite athletes (pp. 77-102). Illinois, United States: Human Kinetics.

Consejo Superior de Deportes (2016). Memoria 2016 de licencias y clubes federados. Disponible en : http://www.csd.gob.es/csd/estaticos/asocfed/LicenciasyClubes-2016.pdf [Consultado el 26 de abril 2018]

Cramer, J.T \& Coburn, J.W (2004). Fitness testing protocols and norms. En R.W. Earle \& T.R. Baechle (Ed.). NSCA's Essentials of Personal Training (pp. 220-263). Illinois, United States: Human Kinetics.

Di Cagno, A., Baldari, C., Battaglia, C., Brasili, P., Merni, F., Piazza, M., ... Guidetti, L. (2008a). Leaping ability and body composition in rhythmic gymnasts for talent identification. The Journal of Sports Medicine and Physical Fitness, 48(3), 341-6. Recuperado de: http://www.ncbi. nlm.nih.gov/pubmed/18974720

Di Cagno, A., Baldari, C., Battaglia, C., Guidetti, L., \& Piazza, M. (2008b). Anthropometric characteristics evolution in elite rhythmic gymnasts. Italian Journal of Anatomy and Embryology, 113 (1), 29. Recuperado de: https://europepmc.org/abstract/med/18491452

Di Cagno, A., Baldari, C., Battaglia, C., Monteiro, M. D., Pappalardo, A., Piazza, M., \& Guidetti, L. (2009). Factors influencing performance of competitive and amateur rhythmic gymnastics-Gender differences. Journal of Science and Medicine in Sport, 12 (3), 411-416. doi:10.1016/j. jams.2008.01.006

Di Cagno, A., Battaglia, C., Fiorilli, G., Piazza, M., Giombini, A., Fagnani, F., .. Pigozzi, F. (2014). Motor learning as young gymnast's talent indicator. Journal of Sports Science and Medicine, 13(4), 767-773. Recuperado de: https://www.ncbi.nlm.nih.gov/pmc/articles/PMC4234945/

Donti, O., Bogdanis, G. C., Kritikou, M., Donti, A., \& Theodorakou, K. (2016). The relative contribution of physical fitness to the technical execution score in youth rhythmic gymnastics. Journal of Human Kinetics, 51(1), 143-152. doi:10.1515/hukin-2015-0183

Donti, O., Tsolakis, C., \& Bogdanis, G. C. (2014). Effects of baseline levels of flexibility and vertical jump ability on performance following different volumes of static stretching and potentiating exercises in elite gymnasts. Journal of Sports Science and Medicine, 13(1), 105-113. Recuperado de: https://www.ncbi.nlm.nih.gov/pubmed/24570613

Douda, H. T., Toubekis, A. G., Avloniti, A. A., \& Tokmakidis, S. P. (2008). Physiological and anthropometric determinants of rhythmic gymnastics performance. International Journal of Sports Physiology and Performance, 3(1), 41-54. doi:10.1123/ijspp.3.1.41

Faulkner J.A (1968). Physiology of swimming and diving. En: Falls H (Ed.) Exercise physiology. Baltimore: Academic Press.

Féderation Internationale de Gimnastique. (2017). Código de Puntuación Gimnasia Rítmica 2017-2020. Disponible en: http://aragongym. com/2016/NORMATIVA/RG_CoP\%202017-2020_sp.pdf [Consultado el 22 de marzo 2018]

Guidetti, L., Baldari, C., Capranica, L., Persichini, C., \& Figura, F. (2000). Energy cost and energy sources of ball routine in rhythmic gymnasts. International Journal of Sports Medicine, 21(3), 205-9. doi:10.1055/s-2000-8879

Hume, P. A., Hopkins, W. G., Robinson, D. M., Robinson, S. M., \& Hollings, S. C. (1993). Predictors of attainment in rhythmic sportive gymnastics. The Journal of Sports Medicine and Physical Fitness, 33(4), 36777. Recuperado de: http://www.ncbi.nlm.nih.gov/pubmed/8035585

Lockie, R. G., Schultz, A. B., Callaghan, S. J., Jeffriess, M. D., \& Luczo, T. M. (2014). Contribution of leg power to multidirectional speed in field sport athletes. Journal of Australian Strength and Conditioning, 22,16-24. doi:10.1071/PP97167

Malina, R. M., Baxter-Jones, A. D. G., Armstrong, N., Beunen, G. P., Caine, D., Daly, R. M., ... Russell, K. (2013). Role of intensive training in the growth and maturation of artistic gymnasts. Sports Medicine (Auckland, N.Z.), 43(9), 783-802. doi:10.1007/s40279-013-0058-5

Purenović-Ivanović, T., \& Popović, R. (2014). Somatotype of top-level serbian rhythmic gymnasts. Journal of Human Kinetics, 40, 181-7. doi:10.2478/hukin-2014-0020

Romero, B., Palomino, A., \& González, J. J. (2011). El perfil antropométrico de la gimnasia rítmica. Apunts Educación Física y Deportes, 103(March 2011), 48-55. Recuperado de: http://www.revista-apunts. com/es/hemeroteca?article $=1465$

Rutkauskaite R \& Skarbalius A. (2011). Interaction of training and performance of 13-14 year old athletes in rhythmic gymnastics. Ugdumas Kuno Kultura, 82, 29-36

Sinning, W. E. (1978). Anthropometric estimation of body density, fat, and lean body weight in women gymnasts. Medicine and Science in Sports, 10(4), 243-9. Recuperado de: http://www.ncbi.nlm.nih.gov/ pubmed/750841

Sociedad Internacional para el Avance de la Cineantropometría (2005). Normas internacionales para la valoración antropométrica. Recuperado de: https://antropometriafisicaend.files.wordpress.com/2016/09/ manual-isak-2005-cineantropometria-castellano1.pdf

Stanton, R., Wintour, S. A., \& Kean, C. O. (2017). Validity and intra-rater reliability of MyJump app on iPhone $6 \mathrm{~s}$ in jump performance. Journal of Science and Medicine in Sport, 20(5), 518-523. doi:10.1016/j. jsams.2016.09.016

Young, W. (1995). Laboratory strength assessment of athletes. New Studies in Athletics, 10, 89-89. 